\title{
MICROBIOLOGÍA DE LAS LESIONES APICALES CRÓNICAS SUPURATIVAS CON FÍSTULA
}

\author{
Liliana Arbeláez ${ }^{i}$ \\ Sandra Patricia Bravo ${ }^{2}$ \\ Carolina Chica ${ }^{l}$ \\ Adolfo Contreras $^{2}$ \\ Adriana Marcela Ospina ${ }^{3}$ \\ Lina Maria Renteria ${ }^{3}$ \\ Luis Felipe Uribe ${ }^{3}$
}

Palabras claves: Periodontitis apical, fístula, microbiota endodóntica

\section{RESUMEN:}

Se identificó la microbiota de 24 dientes que presentaron Periodontitis Apical Crónica Supurativa con lesión fistulosa de 48 muestras de pacientes que asistieron a las clínicas integrales del adulto de la Escuela de Odontología de la Universidad del Valle. Se tomaron muestras de la lesión periapical por el conducto con lima 08 Maillefer $B$ sobrepasando $2 \mathrm{~mm}$ el ápice radiográfico; y de la fistula con puntas de papel estériles \# 15 Meta Dental $\mathrm{Co} \$$ previa preparación del diente y desinfección de la mucosa adyacente al tracto fistuloso. Las muestras microbiológicas fueron colocadas en un medio de transporte VMGA III , procesadas en las 24 horas siguientes, cultivadas y analizadas observando las características de la colonia. Hubo un crecimiento bacteriano de 18 especies, los microorganismos más frecuentes en el conducto fueron Streptococcus anaerobios y Fusobacterium $S p(20.8 \%) 5$ casos, seguidos de Prevotella intermedia y Peptostreptococcus micros (16.7\%) 4 casos, bacilos entéricos gram () y Eubacterium Sp (12,5\%) 3 casos, respectivamente. En la muestra microbiológica de la fistula los microorganismos más frecuentes fueron Porphyromonas gingivalis (33.3\%) 8 casos, Streptococcus anaerobios $(29.2 \%) 7 \mathrm{ca}-$ sos, Peptostreptococcus micros ( $25 \%$ ) 6 casos. La presencia de bacterias patógenas en la lesión perirradicular y el tracto fistuloso pueden ser prevalentes según lo demuestra este estudio. La determinación de la microbiota endodóntica en nuestro medio podría servir para complementar la terapia mecánica con tratamiento antibiótico.

\footnotetext{
Odontóloga Unîversidad del Valle

" Profesor titular escuela de Odontologia

${ }^{3}$ Estudiantes décimo semestre Odontologia Universidad del Valle
} 


\section{INTRODUCCIÓN:}

La Periodontitis Apical usualmente aparece como una lesión inflamatoria crónica que puede ocurrir concomitantemente con síntomas clínicos que incluyen: dolor, sensibilidad apical a la palpacióny percusión e inflamación. En algunos casos, los sintomas pueden estar ausentes. ${ }^{1}$ Estudios recientes sugieren que algunas especies bacterianas, especialmente, Fusobacterium nucleatum, Porphyromonas gingivalis, Porphyromonas endodontalis y Prevotella melaninogenica, están relacionadas con síntomas y signos clínicos propios de esta patología ${ }^{2,3,4}$.

Las bacterias expresan algunos factores que le permiten evadir la respuesta de defensa del huésped. Especies de Prevotella, Porphyromonas, Eikenella y Peptostreptococcus poseen cápsulas bacterianas ${ }^{1}$. Las proteasas producidas por numerosas especies pueden destruir tanto inmunoglobulinas como otras proteínas del sistema de defensa del huésped ${ }^{1}$. Algunas endotoxinas (lipopolisacaridos) producidas por las bacterias gram (-) pueden jugar un papel importante en la reacción periapical y la resorción ósea, probablemente como resultado de mecanismos indirectos que activan la respuesta inflamatoria (sistema del complemento y prostaglandinas) y por activación directa de osteoclastos ${ }^{1}$. Las proteasas (colagenasas, fibrinolisinas y otras que interactúan con inmunoglobulinas y con las proteinas del tejido conectivo) son producidas por especies de bacterias proteolíticas como: Prevotella, Porphyromonas, Peptostreptococcus, Fusobacterium y Enterococcus. Hialuronidasa. condroitin sulfatasa, glucuronidasa, DNAsas, y otras son producidas por especies de Streptococcus, Propionibacterium, Prevotella, Porphyromonas y Fusobacterium ${ }^{3}$.

Debido a la naturaleza polimicrobiana de la flora del canal radicular, la capacidad total de los grupos bacterianos es mayor que cuando existe una sola especie bacteriana!
El cuadro histológico de estos procesos inflamatorios no es uniforme, pero comparte algunas características comunes. Básicamente, la reacción periapical puede ser descrita como tejido granular con tres zonas separadas: las áreas cercanas al ápice contienen grandes cantidades de linfocitos B y T, mientras que las áreas más periféricas son casi carentes de linfocitos pero presentan colágeno y fibroblastos con osteoclastos adyacentes al tejido óseo (signo de pérdida ósea o remodelación). Los granulocitos neutrófilos y los macrófagos se encuentran frecuentemente en la zona rica en células e indican una forma más aguda de infección; se han encontrado granulocitos basófilos y eosinófilos, lo que sugiere reacciones tisulares aún más complejas. ${ }^{1}$

Las infecciones odontogénicas, como otras infecciones en el cuerpo, siguen la via de menor resistencia. La formación y ubicación de tractos sinuosos en infecciones endodónticas está determinada por varios factores: la orientación del ápice radicular, la relación del sitio de perforación a las inserciones musculares en maxila y mandíbula y el grosor de la tabla ósea ${ }^{5}$.

Según el primer estudio nacional de morbilidad oral realizado entre 1977 y 1980 la prevalencia de las patologías diagnosticadas como absceso dental fue de $0.6 \%$ a nivel nacional ${ }^{6}$.

El tercer Estudio Nacional de Salud Bucal ENSAB III y el Segundo Estudio Nacional de Factores de Riesgo de Enfermedad Crónica ENFREC II realizado en 1998 reporta que la prevalencia de absceso dentario con presencia de fistula en tejido blando es del $3.3 \%$ y sin fistula de $1.1 \%$.

La microbiologia de estas lesiones ha sido clarificada a medida que las técnicas de estudio han evolucionando. Inicialmente se pensó que las lesiones periapicales eran medios estériles? ${ }^{7}$. Miller en 1894 fue el primero en sugerir que las bacterias estaban involucradas en la enfermedad pulpar y perirradicular ${ }^{4}$. Con el fin de destacar la importancia de las bacterias, Kakehashi y col. 
expusieron las pulpas dentales de ratas convencionales y ratas libres de gérmenes a su propia microbiota, lo que originó el desarrollo de lesiones pulpares y periapicales en las ratas convencionales, pero, no en las ratas libres de gérmenes ${ }^{8}$. En la década de los 70, se consideraba que la microbiota de los conductos radiculares infectados estaba compuesta por bacterias facultativas ${ }^{9}$; estudios más recientes han demostrado una microbiota anaerobia mixta predominante. Las especies de Bacteroides negros pigmentados han adquirido especial relevancia en la investigación de agentes etiológicos asociados con las infecciones endodónticas ${ }^{10,11,12}$.

En 1992, Sundqvist et al, ${ }^{3}$ realizó un estudio donde la mayor parte de las bacterias fueron anaerobios estrictos y aisló en promedio cinco cepas bacterianas por conducto radicular, así: Eubacterium spp (59 cepas), Peptostreptococcus spp ( 54 cepas), Fusobacterium spp (50 cepas), Porphyromonas spp (32 cepas), Prevotella spp pigmentadas (30 cepas), Streptococcus spp ( 28 cepas), Wolinella spp (18 cepas), Prevotella spp no pigmentadas (15 cepas), Actinomyces spp (14 cepas), Propionibacterium spp y Capnocylophaga ochracea 7 cepas, Veillonella párvula y Selenomonas sputigena 6 cepas respectivamente.

Las muestras microbiologicas fueron tomadas de 24 dientes a $5 \mathrm{~mm}$ del foramen apical de los conductos radiculares infectados en dientes con $\mathrm{Pe}$ riodontitis Apical Crónica Supurativa .

Gary V. Vigil et al, ${ }^{13}$ estudiaron la microbiología de lesiones periapicales con fistula y sin fistula y encontraron que las especies predominantes en lesiones sin fistula fueron:

Propionibacterium acnes, Staphylococcus epidermidis y Streptococcus intermedius. En lesiones con fistula, las especies encontradas fueron: Wolinella recta, Eubacterium lentum, Porphyromonas gingivalis, Fusobacterium sp y Clostridium sp.
El papel fundamental de las bacterias en la inducción de las lesiones apicales ha estado bien documentado en estudios tanto de animales como humanos. Los hallazgos de Kakehashi et al, en 1965 y Sundqvist en 1976 establecieron claramente que las enfermedades endodónticas dependen de la presencia de microorganismos 4 .

De los cientos de especies microbianas que colonizan la cavidad oral, algunas bacterias han sido involucradas en la patogénesis de lesiones perirradiculares. Estudios han mostrado que especies de los géneros Fusobacterium, Prevotella, Porphyromonas, Eubacterium, Peptostreptococcus, Actinomyces y Propionibacterium se encuentran con mayor prevalencia en infecciones primarias de canales radiculares ${ }^{14}$.

La composición microbiana de un canal radicular infectado está determinada por la ruta mediante la cual la bacteria gana acceso al conducto y por factores ecológicos. Diferentes especies de microorganismos pueden alcanzar el conducto radicular de forma casual si existe una comunicación abierta a la cavidad oral y su microbiota. Por tanto, la probabilidad de encontrar una gran variedad de especies bacterianas orales es mayor en lesiones abiertas que si la infección ha ocurrido a través de mecanismos más selectivos como lesiones cariosas recurrentes, infección de los túbulos dentinales, fracturas radiculares o coronales o difusión hematógena ${ }^{1}$.

El conocimiento de la dinámica de la microbiota del canal radicular ha sido clarificado a través de experimentos en 52 canales radiculares autoinfectados y experimentalmente

lacerados en monos, realizados por Fabricius et al, ${ }^{15}$. Inicialmente especies facultativas de Streptococcus, Enterococcus, Lactobacillus, Corynebacterium, $\mathrm{y}$ bastones coliformes fueron tan frecuentes como los anaerobios: Peptostreptococcus, Propionibacterium, Eubacterium, Prevotella y Fusobacterium. La proporción relativa de especies anaeróbicas aumentó con el tiempo mientras que la proporción de especies facultativas disminuyó y finalmente fue casi nula' ${ }^{3}$. 
Aunque las bacterias han sido las más estudiadas, especies de levaduras tambiẻn pueden estar asociadas con canales radiculares infectados. La presencia de Candida albicans fue detectada en 5 de $24(21 \%)$ muestras tomadas de canales radiculares ${ }^{16}$. Así mismo, estudios recientes asocian la presencia de levaduras con infecciones resistentes a la terapia endodontica y antibiótica ${ }^{16}$. Las levaduras son consideradas normales en cavidad oral, pero, pueden producir enfermedades cuando hay factores predisponentes locales 0 sistémicos para la infección ${ }^{16}$.

Según estudios de susceptibilidad antibiótica ${ }^{2}, 20$ especies bacterianas fueron recuperadas en 15 casos ( 14 de 15 casos en este estudio tuvieron algún retratamiento). Las especies más comúnmente detectadas fueron: Streptococcus a hemolíticos y Enterococcus. Los resultados de la prueba de sensibilidad antibiótica revelaron que el Enterococcus sp fue altamente resistente a los antibióticos, especialmente a las cefalosporinas ${ }^{2}$ .El Enterococcus sp está presente en infecciones oportunistas que involucran la cavidad oral; éste se encuentra conmúnmente en el intestino humano pero también puede encontrarse de manera temporal en la cavidad oral. Especialmente, el Enterococcus faecalis ha sido identificado en placa bacteriana, saliva, superficie mucosa y encía ${ }^{17}$. La presencia de Enterococcus en la cavidad oral ha sido asociada con lesiones de mucosa en pacientes inmunocomprometidos e infecciones radiculares persistentes ${ }^{17}$.

De las 500 especies bacterianas presentes en la cavidad oral, solo un número relativamente bajo de especies se encuentran en el canal radicular, indicando que presiones selectivas actúan en dicho canal ${ }^{18}$. Las asociaciones microbianas entre especies han sido determinadas analizando la posibilidad de detectar unas especies en presencia o . ausencia de otras. Se encontraron asociaciones bacterianas positivas muy fuertes entre ciertas especies asi: Fusobacterium nucleatum fue asociado positivamente con Peptostreptococcus micros, Campylobacter rectus, Porphyromonas (antiguamente Bacteroides) endodontalis, y Selenomonas sputigena. Bacteroides intermedius fue asociado positivamente con: Peptostreptococcus anaerobius, P. micros y Eubacterium sp. Hubo también una asociación positiva fuerte entre Eubacterium alactolyticum y $P$. anaerobius. Hay varios factores que pueden influenciar el crecimiento y colonización de bacterias en los canales radiculares ${ }^{18}$. La disponibilidad de nutrientes, la baja tensión de oxígeno en canales radiculares con pulpas necróticas y la interacciones bacterianas pueden ser determinantes ecológicos importantes ${ }^{19}$.

En un estudio realizado en la Universidad Estació de Sá de Rio de Janeiro, que analizó la microbiota de 28 canales radiculares infectados, se demostró la presencia y niveles significativos de 42 especies bacterianas por medio del método de la tarjeta de identificación de análisis de hibridización genómica DNA-DNA; así mismo demostró que 22 de las 42 especies de DNA probadas fueron reactivas con una o más muestras. Las especies más prevalentes fueron: Bacteroides forsythus $(39.3 \%)$, Haemophilus aphrophilus $(25 \%)$, Corynebacterium matruchotti (21.4\%), Porphyromonas gingivalis $(17.9 \%)$ y Enterococcus faecalis, Capnocytophaga gingivalis y Streptococcus intermedius (14.3\%) respectivamente Los hallazgos más inusuales de especies fueron: Ralstonia sp. y Pseudomona aeruginosa, en pocos casos y en porcentajes del cultivo pequeños. La discusión que surgió de los hallazgos se basa en la presencia y alta prevalencia de cierto tipo de bacterias que nunca habian sido reportadas en otros estudios con métodos de cultivo tradicionales, como es el caso de Haemophilus aphropilus, Corynebacterium matruchotii y Treponema dentícola. Por otra parte algunas especies bacterianas comúnmente encontradas en infecciones endodónticas mediante cultivo como son: Actinomyces sp, $P$, anaerobius, $P$. nigrescens, $P$. acnes y algunas especies usuales de Streptococcus orales no se detectaron en esta investigación ${ }^{14}$. 


\section{MATERIALES Y METODOS:}

Con el fin de determinar la microbiota cultivable en infecciones endodónticas y dar un enfoque adecuado al tratamiento, se realizó un estudio descriptivo en 24 pacientes con Periodontitis Apical Crónica Supurativa (P.A.C.S) que consultaron las clinicas Integrales del Adulto de la Universidad del Valle entre Enero del 2001 a Abril del 2002, con criterios de inclusión y de exclusión previamente establecidos. Los participantes fueron escogidos por conveniencia de acuerdo con las características médicas y odontológicas establecidas para el estudio (determinación no probabilística del tamaño de la muestra). Para la toma de las muestras fue necesaria una recopilación de los datos clínicos del paciente (historia clinica) y una radiografia periapical que confirmó el diagnóstico clínico.

El estudio siguió las recomendaciones de los comités de ética humana de la facultad de salud de la Universidad del Valle y lo planteado en la resolución 8430 para protección de sujetos humanos en investigación biomédica. Se explicó a cađa paciente el tipo de procedimiento a realizar, además se efectuó un examen periodontal; los pacientes firmaron un formato de aceptación y participación voluntaria en el estudio.

Los criterios de inclusión que se tuvieron en cuenta fueron: pacientes mayores de 6 años de edad, todos los dientes estudiados fueron de dentición permanente, con signos clínicos y radiográficos de Periodontitis Apical Crónica Supurativa; dientes con destrucción coronal por caries, trauma o yatrogenia, con fistula; $y$ sin afección periodontal. Se seleccionaron pacientes que no hubieran tomado antibióticos en los últimos 3 meses para evitar modificación en la microbiota endodóntica y en la microbiota de la fistula. Entre los criterios de exclusión se tuvieron en cuenta: pacientes que presentaron Periodontitis Apical Aguda; ausencia de fistula; pacientes embarazadas o comprometidos sistémicamente (diabetes, VIH-SIDA, enfermedades infectocontagiosas agudas y enfermedades hereditarias); pacientes con malforma- ciones dentales de estructura (hipoplasia del esmalte, dentinogénesis imperfecta, amelogénesis imperfecta, etc...) y lesiones endodónticas en dientes temporales.

Se tomaron muestras de pacientes de sexo femenino y masculino de procedencia rural y urbana. Como parámetros educativos se tuvieron en cuenta los niveles de educación: primaria, secundaria y universitaria. En cada paciente se tomó una radiografia periapical y además se realizó sondaje (sonda periodontalHu-Friedyâ milimetrada) para evaluar la profundidad del surco periodontal, la cual siempre estuvo entre 0 y $3 \mathrm{~mm}$.

A los pacientes seleccionados se les realizó una historia clínica que incluyó: identificación del paciente, anamnesis (antecedentes personales y familiares médicos generales y odontológicos); conductometria tentativa y sobrepasada en $2 \mathrm{~mm}$ para la toma de los microorganismos de la lesión. Se tomó una conductometria tentativa con técnica radiográfica estandarizada (paralelismo) para determinar la longitud apropiada con la que se tomaron las muestras.

Para la muestra del conducto se preparó el diente removiendo placa blanday caries. Luego se procedió al aislamiento total del diente con tela de caucho y se realizó una ambientación cameral para mejorar la accesibilidad al conducto radicular (Foto 3). La corona se limpió con peróxido de hidrógeno al $30 \% 035 \%$ con jeringa hipodérmica de 10 c.c. en repetidas aplicaciones hasta que no hubo formación de burbujas. Posteriormente se aplicó Isodine al 5\% o 1\% y luego una solución de tiosulfato para inactivar la acción del Isodine, $\mathrm{El}$ tercio coronal del conducto radicular se instrumentó con una técnica retrógada y se irrigó con suero fisiológico estéril; se introdujo luego una lima \# 08 nueva y estéril hasta $2 \mathrm{~mm}$ después de sobrepasar el ápice radicular (basados en una conductometría tentativa de acuerdo con la radiografia previa); inmediatamente después la muestra se colocó en el medio de transporte prerreducido VMGA III ${ }^{20}$. 
Para la toma de la muestra por la fistula se preparó la mucosa mediante aislamiento de la zona con algodones estériles en un área de $2 \mathrm{~cm}$ alrededor del orificio en la mucosa; se limpió en repetidas ocasiones con peróxido de hidrógeno; se introdujo una punta de papel impregnada con peróxido de hidrógeno $2 \mathrm{~mm}$ por el orificio de la fístula; se aplicó Isodine alrededor y se inactivó con solución de tiosulfato de sodio, se secó con gasas estériles. Se introdujo una punta de papel estéril por el orificio de la fistula hasta llegar a la lesión más o menos $5 \mathrm{~mm}$ (se mantuvo 8 a 10 segundos); inmediatamente la muestra se colocó en el medio de transporte prerreducido VMGA III ${ }^{20}$.

El análisis microbiológico incluyó un cultivo no selectivo y selectivo de los patógenos anaerobios y facultativos. Los microorganismos fueron mecánicamente dispersados del vial conteniendo VMGA III con un bactovortexâ por 45 segundos. La solución microbiana fue diluída 10 veces hasta concentraciones $10^{-3}, 10^{-5}, 10^{-7}$ para la Brucella agar sangre con solución VMGA I. 0.1 $\mathrm{ml}$ de las diluciones de la muestra fueron puestas en un medio de Brucella agar sangre de cordero no selectivo suplementado con $0.3 \%$ de Bacto agar, $5 \%$ de sangre desfibrinada, $0.2 \%$ de eritrocitos hemolizados de cordero, $0.0005 \%$ de Hemina y $0.00005 \%$ de Menadiona para determinar el número total de colonias y las proporciones de bacterias especificas en relación con el número total de colonias. $0.1 \mathrm{ml}$ de la muestra original y $10^{-1}$ de la dilución de VMGA III se pusieron en un medio (Tripticasa Soya Agar Vancomicina y Bacitracina) TSVB para el cultivo de Actinobacillus actinomicetemcomitans, Bacilos Entéricos Gram (-), Pseudomonas y Levaduras.

El Brucella agar con sangre de cordero fue incubado a $35^{\circ} \mathrm{C}$ en una cámara anaeróbica con un contenido de $85 \%$ de $\mathrm{N}_{2}-10 \mathrm{H}_{2}-5 \% \mathrm{CO}_{2}$ por 7 dias. El medio TSVB fue incubado en $10 \%$ de aire $\mathrm{CO}_{2}$ a $37^{\circ} \mathrm{C}$ por 4 días.
Al término del cual los cultivos se sacaron del incubador y se observó el crecimiento estudiando las características de la colonia, utilizando microscopio esteroscópico Bausch \& Lombâ.

El análisis estadístico usó la prueba " $\mathrm{t}$ " de Student para muestras apareadas comparando dos variables numéricas (microbiología de fistula y conducto). No fue necesario realizar otro tipo de pruebas debido a que el número de muestras microbiológicas estudiadas fue mayor de treinta; cuando la significancia es mayor de 0,5 se asume que la diferencia entre la microbiota del conducto y la fistula no es estadísticamente relevante.

\section{RESULTADOS:}

Se estudió la microbiología de las lesiones Apicales Crónicas Supurativas con proceso fistuloso de 48 muestras en 24 pacientes durante un período de 15 meses en las Clínicas Integrales del Adulto de la Escuela de Odontología de la Universidad del Valle. Se estudiaron 13 hombres y 11 mujeres con un rango de edad entre 21 y 67 años y un promedio de edad de 44,5 años. Los pacientes estudiados provinieron, 3 (12.4\%) de la zona rural y $21(87.6 \%)$ de la zona urbana de Cali. Se estudiaron 13 dientes multirradiculares $(54.16 \%)$ y 11 dientes unirradiculares (45.84\%). Los dientes afectados con mayor frecuencia fueron: incisivos superiores 7 casos $(29.2 \%)$; molares superiores 5 casos $(20.9 \%)$ y primeros molares inferiores y premolares superiores 4 casos $(16.7 \%)$; incisivos inferiores, canino superior, canino inferior y premolares inferiores un caso $(4,2 \%)$. Los procesos fistulosos siempre aparecieron en la tabla ósea vestibular.

El promedio del conteo total de colonias (TCC) en conducto fue de $129.08 \times 10^{5} \mathrm{y}$ el promedio del conteo total de colonias en fistula fue de 103.25 $\times 10^{5}$ con un crecimiento bacteriano de 18 especies. Los microorganismos más frecuentes en el conducto fueron: Streptococcus anaerobios y Fusobacterium sp presentes en 5 de las 24 muestras (20.8\%), seguidos de Prevotella interme- 
dia y Peptostreptococcus micros en 4 casos (16.7\%), respectivamente. Los bacilos entéricos gram (-) y Eubacterium sp aparecieron en 3 casos $(12.5 \%)$. Propionibacterium propionicum (antes Arachnia propionica), Peptostreptococcus anaerobios, Campylobacter sp, Porphyromonas gingivalis y Propionibacterium en 2 casos $(8.3 \%)$. Las especies menos comunes fueron: Enterococcus sp, Bacteroides forsythus, Staphylococcus, Actinomyces, Dialister pneumosintes $y$ Veillonella que aparecieron en un caso (4.2\%), respectivamente. No se evidenció crecimiento de Eikenella corrodens, Actinobacillus actinomicetemcomitans, Streptococcus â hemolificos, ni levaduras en los cultivos del conducto.

En las muestras obtenidas del tracto fistuloso los microorganismos que se presentaron con mayor frecuencia fueron: Porphyromonas gingivalis 8 casos (33.3\%), Streptococcus anaerobios 7 muestras $(29.2 \%)$, seguidos por Peptostreptococcus micros en 6 casos $(25 \%)$. Bacilos entéricos gram(-) en 5 muestras (20.8\%); Eubacterium sp 4 casos (16.7\%); Prevotella intermedia, Peptostreptococcus anaerobios y Fusobacterium sp en 3 muestras $(12.5 \%)$. Dialister pneumosintes y Eikenella corrodens en 2 casos $(8.3 \%)$. Los microorganismos con menor frecuencia de aparición fueron: Propionibacterium propionicus, Campylobacter sp, Actinomyces y Enterococcus sp con 1 caso (4.2\%). No se encontraron Veillonella sp, Staphylococcus sp, ni Bacteroides forsythus. (Tabla 1)

De los 6 casos en donde se determinó la presencia de Bacilos entéricos gram (-), sólo en dos se pudo identificar la especie: Enterobacter gergoviae (un caso) y Hafnia alvei (un caso).

Se presentó un mismo microorganismo en conducto y fistula en 15 casos, asi: Prevotella intermedia, Streptococcus anerobios y Fusobacterium sp 3 casos, respectivamente; Peptostreptococcus micros, Peptostreptococcus anaerobios y Bacilos entéricos en 2 casos (Tabla 2). Debido a que la prueba de significancia para al- gunos microorganismos fue mayor del 0.05 (prueba t-Student), puede concluirse, que estadísticamente no hay diferencia entre la microbiologia del conducto y la microbiología de la fistula.

Se encontró que hubo una presencia positiva de Peptostreptococcus micros en la fistula y baja en el conducto que fue estadísticamente significativa (Tabla $2 \mathrm{t}$-Student $\mathrm{P}<0.001$ ). En contraste, microorganismos tales como bacilos entéricos gram (-) y Peptostreptococcus anaerobios aparecieron en ambas muestras con una diferencia estadísticamente significativa (Tabla $2 \mathrm{t}$-Student $\mathrm{P}<0.001$ )

\section{DISCUSIÓN:}

Este estudio reveló la microbiologia presente en las lesiones Apicales Crónicas Supurativas y en los tractos fistulosos de 24 casos. Con excepción de $\operatorname{los} 6$ casos en donde se cultivaron bacilos entéricos gram (-), la microbiología cultivada correspondió a organismos anaerobios gram $(-)$ en mayoría y pocos anaerobios gram $(+)$ como Peptostreptococcus micros, Peptostrptococcus anaerobios y Staphylococcus. Brook et al, demostró que las bacterias anaerobias son importantes en la formación de abscesos periapicales y otras infecciones supurativas ${ }^{21}$.

Los bacilos entéricos gram (-) al igual que Porphyromonas gingivalis se encontraron con más frecuencia en la fistula que en el conducto, pero esta diferencia no fue significativa.

Los microorganismos encontrados con mayor frecuencia en el conducto radicular fueron: Streptococcus anaerobios, Fusobacterium sp, Peptostreptococcus micros y Prevotella intermedia. Esta información confirma los hallazgos de Sundqvist (1992), donde se aisló: Eubacterium sp (59 cepas), Peptostreptococcus sp (54 cepas), Streptococcus anaerobios (50 cepas), Porphyromonas gingivalis ( 32 cepas), Prevotella sp y Fusobacterium sp ( 30 y 50 cepas respectivamente); además aisló Actinobacillus actinomicetemcomitans, ${ }^{19}$ cuya presencia no fue demostrada en 
nuestro estudio, por tanto no se muestra en las tablas.

Young Jung et al, estudió la epidemiología de patógenos putativos en conductos radiculares con Periodontitis Apical mediante reacción en cadena de la polimerasa (PCR) y pruebas de hibridización, encontrando como especies bacterianas más frecuentes: Fusobacterium sp (68.4\%), Peptostreptococcus micros (44.7\%) y Porphyromonas gingivalis (26.3\%), microorganismos también hallados en este estudio. Young Jung tampoco demostró la presencia de Actinobacillus actinomicetemcomitans ${ }^{18}$. Estudios realizados por Dahlén G. et al, y Peciuliene et al, revelaron la presencia de Enterococcus sp en infecciones endodónticas bajo tratamiento con $\mathrm{Ca}(\mathrm{OH})_{2}$ y dientes con Periodontitis Apical previamente obturados, respectivamente; en nuestro estudio se reporta la presencia en 1 caso (en conducto y fistula) ${ }^{17,22}$.

No se encontraron diferencias estadísticamente significativas en la detección de microorganismos entre el conducto radicular y la fístula.

El total de especies bacterianas aisladas (TCC) tanto del conducto como de la fistula difieren considerablemente un caso del otro, dicha variación puede explicarse por las diferencias en la exposición (en tiempo) al aire en cada muestra, dependiendo de la accesibilidad al sitio de la toma; otros factores importantes que pudieron contribuir a la variabilidad son : la historia dental del curso de la enfermedad y las condiciones ambientales del conducto radicular como son nutrición y presión parcial de oxigeno que pueden tener variaciones en cada caso y los procesos de desinfección inicial del conducto y fístula.

Baumgartner J et al, demostró mediante PCR la presencia de Candida albicans en infecciones de conductos radiculares, detectándola en 5 de 24 $(21 \%)$ muestras ${ }^{17}$; a diferencia del presente estudio, el cual no reveló la presencia de levaduras en ningún caso; aunque esta determinación fue por cultivo.
Este estudio encontró una presencia alta de Peptostreptococcus micros en la muestra microbiológica del conducto y baja en la muestra microbiológica de la fístula, que fue estadisticamente significativa(Tabla 2). Estudios previos demuestran la presencia de este microorganismo en infecciones endodónticas, pulpas necróticas y abscesos periapicales ${ }^{14,18,21}$. Siquiera el al, demostró que $P$. micros fue una de las especies predominantes en nueve dientes estudiados que presentaron sensibilidad a la percusión ${ }^{14}$. Así mismo, este estudio demostró la presencia estadisticamente significativa de Peptostreptococcus anaerobious y bacilos entéricos $\operatorname{gram}(-)$ en ambas muestras microbiológicas (ver Tabla 2). G. Dahlény Å.J.R. Möller encontraron que Peptostreptococcus constituye el segundo género más común $(18 \%)$ de los aislados en abscesos periapicales; siendo $P$. anaerobious, $P$. micros, $P$. prevotii y $P$. magnus las especies más frecuentes!.

No se cultivaron organismos aerobios como Staphylococcus sp probablemente porque el proceso de desinfección de conducto y fístula inicial inactivaron o eliminaron estos microorganismos 0 porque no se emplearon medios no selectivos en condiciones aeróbicas que permitan el cultivo de estos organismos.

La presencia de bacterias patógenas en la lesión perirradicular y el tracto fistuloso fueron prudentes según lo demuestra este estudio. La determinación de la microbiota endodóntica en nuestro medio sirve para complementarla con antibióticos. Así mismo, debido a que la Periodontitis Apical Crónica Supurativa (P.A.C.S) recurrente se puede deber a la persistencia y /o virulencia de bacterias en la lesión periapical se hace necesario un tratamiento que incluya estudios microbiológicos y de sensibilidad antibiótica complementando la terapia mecánica. 


\section{SUMMARY:}

The microbiology of 24 teeth with Suppurative Chronic Apical Periodontitis and fistula lesions were identified. Forty eight samples were taken from adult patients that attended to the Dental School at the Universidad del Valle, Cali-Colombia. Root canal samples were taken after crown drilling and disinfection which included also the adjacent mucous of the fistulous tract. Root canal samples were recovered with a 08 Maillefer $\mathbb{R}$ sterile file overstepping $2 \mathrm{~mm}$ in the apical lesion. Fistula samples were recovered using \# 15 Meta Dental $\mathrm{Co} 8$ sterile paper points. Both, fistula and root canal samples were transfered to VMGA III transport media and processed at least 24 hours after sampling. Plating took place at the Oral Microbiology laboratory using selective media such as TSVB and non-selective media such as BBL plates supplemented with $5 \%$ sheep blood/hemin adn menadione. Anaerobic and facultative cul- ture were incubated for 5 days. Microbiological determination was done using stereostopic microscopy for microbial colony morphology and rapid biochemical test. This study identified eighteen bacteria species. The more frequent species in root canals were: Streptococcus anaerobios and Fusobacterium sp (20.8\%) 5 cases, Prevotella intermedia and Peptostreptococcus micros (16.7\%) 4 cases, Eubacterium sp and Gram () enteric rods (12.5\%) 3 cases. The more frequent species in the fistula samples were: Porphyromonas gingivalis (33.3\%) 8 cases, Streptococcus anaerobios (29.2\%) 7 cases, Peptostreptococcus micros (25\%) 6 cases. According to this results, the presence of pathogenic bacterias in the periapical lesion and in the fistulous tract may be important because the determination of endodontic microbiolgy would be useful to complement the mechanical therapy with antibiotic treatment.

\begin{tabular}{|c|c|c|c|c|}
\hline TIPO DE BICHOORGANESWO & $\begin{array}{l}\text { FHECUENCA } \\
\text { CONDUCTO }\end{array}$ & $\begin{array}{l}\text { PORCENTANE } \\
\text { CONDUCTO }\end{array}$ & $\begin{array}{l}\text { FRECUERCSA } \\
\text { FISTULA: }\end{array}$ & $\begin{array}{l}\text { POACEGTINE } \\
\text { RistuLA }\end{array}$ \\
\hline Proveltita intermoda & 4 & 18.7 & 3 & 125 \\
\hline Fimpionbacterium prepionicum & 2 & 2.5 & 1 & 42 \\
\hline Sifeptococeus anserobios & 5 & 208 & 7 & 22.2 \\
\hline Vellanella & 1 & 42 & 8 & $\sigma$ \\
\hline Peptostreptococess itherios & 4 & 16.7 & 6 & 25 \\
\hline Peptostroptoconcus anaerobias & 2 & 8.3 & 3 & 12.8 \\
\hline Canpylabacter sq? & 2 & 8.3 & 1 & 4.2 \\
\hline Draister pneumosintas & 1 & 42 & 2 & 83 \\
\hline Fusabacterium so & s & $20: 8$ & 3 & 12.5 \\
\hline Actinantyces & 3 & 42 & 2 & 42 \\
\hline Stephiococoss & 1 & 42 & 8 & 8 \\
\hline Fophyomenas gingivais & 2 & 83 & 8 & 283 \\
\hline Exacterbides forymus & 1 & 42 & Q & 8 \\
\hline Eubacterum sp & 3 & 12.5 & 4 & 167 \\
\hline Ekenefla carrodins: & 0 & 0 & 2 & 8.3 \\
\hline Entenococens sp & 1 & 4.2 & 1 & 42 \\
\hline Propichicactanum & 2 & 8.3 & 0 & 6 \\
\hline Bacliss antencos gram in & 3 & 12.6 & 5 & $20: 3$ \\
\hline $\begin{array}{l}\text { Muestra }(n)=24 \\
\text { Conteo promedio total de coiat }\end{array}$ & $\begin{array}{l}\text { Oitucion } 10^{2} \\
\text { s (TCC) en cen }\end{array}$ & $=129.08 \times 10^{4}$ & & \\
\hline
\end{tabular}


isolated from infected root canals after varied times of closure. Scand J Dent Res. 90:134-44(2), 1982.

J, CRAIG BAUMGARTNER;CHAD, M WATTS: TIAN, XIA. Ocurrence of Candida albicans in infections of endodontic origin. J Endodontics. 26:695$698(12), 2000$.

17DAHLEN, G; SAMUELSSON, W; MOLANDER, A; REIT,C. Identification and antimicrobial susceptibility of Enterococci isolated from the root canal. Oral Microbiol Immunol. 15:309-312(5),2000.

JUNG,Y; et al. Molecular Epidemiology and Association of Putative Pathogens in Root Canal Infection. J Endodontics. 26:599-604(10),2000. anaerobic microbiology of periapical abscess. Oral Microbiol Immunol. 6:123-125(2), 1991.

PECIULIENE, BALCIUNIENE I; ERIKSEN, $\mathrm{H}_{\text {; ,HAAPASALO M. Isolation of Enterococcus }}$ faecalis in Previously Root-Filled Canals in a Lithuanian Population. J Endodontics. 26:593-595(10),2000.

\section{Correspondencia:}

Adolfo Contreras

escuadon@univalle.edu.co

Universidad del Valle

Cali 